\title{
On the adjacent vertex distinguishing proper edge colorings of several classes of complete 4-partite and 5-partite graphs
}

\author{
Xiang-en Chen, Chunyan Ma, Fang Yang, Bing Yao \\ College of Mathematics and Statistics \\ Northwest Normal University \\ Lanzhou, 730070, China \\ e-mail: chenxe@nwnu.edu.cn \\ e-mail: bjau2008ok@163.com
}

\begin{abstract}
A proper $k$-edge coloring of a graph $G$ is an assignment of $k$ colors, $1,2, \cdots, k$, to edges of $G$. For a proper edge coloring $f$ of $G$ and any vertex $x$ of $G$, we use $S(x)$ denote the set of the colors assigned to the edges incident to $x$. If for any two adjacent vertices $u$ and $v$ of $G$, we have $S(u) \neq S(v)$, then $f$ is called the adjacent vertex distinguishing proper edge coloring of $G$ (or AVDPEC of $G$ in brief). The minimum number of colors required in an AVDPEC of $G$ is called the adjacent vertex distinguishing proper edge chromatic number of $G$, denoted by $\chi_{a}^{\prime}(G)$. In this paper, adjacent vertex distinguishing proper edge chromatic numbers of several classes of complete 4-partite and 5-partite graphs are obtained. (Abstract)
\end{abstract}

Keywords-complete 4-partite graphs; complete 5-partite graphs; proper edge coloring; adjacent vertex-distinguishing proper edge coloring (key words)

\section{INTRODUCTION}

The graph coloring has a wide range of applications in real life. In [1]-[6], the vertex distinguishing proper edge coloring of graphs is introduced and investigated; In [7], the adjacent vertex distinguishing proper edge coloring of graphs is introduced and investigated and the adjacent vertex distinguishing proper edge chromatic number of a graph $G$ has been obtained for certain graphs, such as cycles, complete graphs. We use the usual notation as can be found in any book on graph theory, see e.g.[9].

Let $G$ be a simple finite graph with maximum degree $\Delta(G), k$ be a positive integer and $f$ be an edge coloring (i.e., $f$ be an assignment of $k$ colors, $1,2, \cdots, k$, to the edges of $G$ ). For each element $z \in E(G)$, we use $f(z)$ denote the color of z. For every vertex $x \in V(G)$ the set of colors of edges incident with $x$ is denoted by $S(x)$ and is called the color set of $x$. If $f$ is proper and $S(u) \neq S(v)$ for each edge $u v \in E(G)$, then $f$ is called a $k-$ adjacent vertex distinguishing proper edge coloring of $G$ (or a $k$-AVDPEC). The minimum number of colors required in an AVDPEC of $G$ is called the adjacent vertex distinguishing proper edge chromatic number of $G$, denoted by $\chi_{\mathrm{a}}^{\prime}(G)$.
Let $G(V, E)$ be a simple graph and the vertex set $V(G)$ can be partitioned into $n$ stable sets $V_{1}, V_{2}, \cdots, V_{n}$, where $V_{i}=$ $\left\{v_{i j} \mid j=1,2, \cdots, m_{i}\right\}, m_{i}=\left|V_{i}\right|, i=1,2, \cdots, n$. If each vertex in $V_{i}$ is adjacent to any one vertex in $V_{j}$, where $i \neq j, i, j=1,2, \cdots, n$, then $G$ is called a complete $n$-partite graph and denoted by $K_{m_{1}, m_{2}, \cdots, m_{n}}$.

Lemma 1.1 and Lemma 1.2 are obvious.

Lemma 1.1 For any graph $G$ that has no isolated edge and at most 1 isolated vertex, we have $\chi_{\mathrm{a}}^{\prime}(G) \leqslant \chi_{\mathrm{s}}^{\prime}(G)$.

Lemma 1.2 If $m \geqslant n \geqslant p \geqslant q \geqslant 1$, then $\Delta\left(K_{m, n, p, q}\right)=$ $m+n+p$.

Lemma $1.3^{[7]}$ Let $G$ be a simple connected graph with order at least 3 , if $G$ has 2 adjacent vertices of maximum degree, then $\chi_{\mathrm{a}}^{\prime}(G) \geqslant \Delta(G)+1$.

Lemma 1.4 ${ }^{[10]}$ Let $G[\{v \mid d(v)=\Delta(G)\}]$ be an induced subgraph by all vertices of maximum degree in $G$. If $G[\{v \mid$ $d(v)=\Delta(G)\}]$ is a forest, then $\chi^{\prime}(G)=\Delta(G)$.

In [8] Zhao Xinmei et al have obtained the adjacent vertex distinguishing proper edge chromatic numbers of some complete 4-partite graphs. The results in [8] are listed in the following table:

TABLE1: The adjacent vertex distinguishing proper edge chromatic numbers of some complete 4-partite graphs given in [8]

\begin{tabular}{|c|c|c|}
\hline graph $G$ & condition & $\chi_{\mathrm{a}}^{\prime}(G)$ \\
\hline$K_{m, n, p, q}$ & $m>n>p>q \geqslant 1$ & $m+n+p$ \\
$K_{n, 1,1,1}$ & $n \geqslant 2$ & $n+3$ \\
$K_{m, n, 1,1}$ & $m>n \geqslant 2$ & $m+n+2$ \\
$K_{n, n, n, p}$ & $n \geqslant p+2$ and $p \geqslant 2$ & $3 n$ \\
$K_{n, n, p, p}$ & $n \geqslant p+1$ and $p \geqslant 1$ & $2 n+p+1$ \\
$K_{n, p, p, p}$ & $n \geqslant p+2$ and $p \geqslant 2$ & $n+2 p+1$ \\
$K_{n, n, n, n-1}$ & $n \geqslant 2$ & $3 n$ \\
$K_{n, n-1, n-1, n-1}$ & $n \geqslant 3$ & $3 n-1$ \\
\hline
\end{tabular}

Based on the above work we will obtain the adjacent vertex distinguishing proper edge chromatic numbers of other several classes of complete 4-partite and 5-partite graphs in this paper. 


\section{MAin RESUlts}

Suppose $k, l$ are integers, $l \geqslant 1$, we use symbol $(k)_{l}$ to denote the number in $\{1,2, \cdots, l\}$ which is congruent to $k$. For example, $(-3)_{8}=5,(9)_{8}=1,(20)_{8}=4$.

Theorem 2.1 If $m \geqslant n>p \geqslant 1$ and $m+p \leqslant 2 n$, then $\chi_{\mathrm{a}}^{\prime}\left(K_{m, n, n, p}\right)=m+2 n$.

Proof Obviously we have $\chi_{\mathrm{a}}^{\prime}\left(K_{m, n, n, p}\right) \geqslant \Delta\left(K_{m, n, n, p}\right)=$ $m+2 n$. In order to prove $\chi_{\mathrm{a}}^{\prime}\left(K_{m, n, n, p}\right)=m+2 n$, we need only to give the $(m+2 n)$-AVDPEC of $K_{m, n, n, p}$. Suppose the colors that we will use are $1,2, \cdots, m+2 n$.

Let edge $v_{4 i} v_{3 j}$ receive color $(i+j-1)_{m+2 n}$ and edge $v_{4 i} v_{2 j}$ receive color $(n+i+j-1)_{m+2 n}, i=1,2, \cdots, p ; j=$ $1,2, \cdots, n$.

Let edge $v_{4 i} v_{1 j}$ receive color $(2 n+i+j-1)_{m+2 n}, i=$ $1,2, \cdots, p ; j=1,2, \cdots, m$.

Let edge $v_{3 i} v_{2 j}$ receive color $(m+p+i+j-1)_{m+2 n}$, $i, j=1,2, \cdots, n$.

Let edge $v_{3 i} v_{1 j}$ receive color $(p+i+j-1)_{m+2 n}$ and edge $v_{2 i} v_{1 j}$ receive color $(m+n+p+i+j-1)_{m+2 n}, i=$ $1,2, \cdots, n ; j=1,2, \cdots, m$.

We construct a cycle $C$ such that vertex set of $C$ is $m+2 n$ colors $\{1,2, \cdots, m+2 n\}$ and edge set of $C$ is $\left\{(i)_{m+2 n}(i+\right.$ 1) $m+2 n \mid i=1,2, \cdots, m+2 n\}$.

The color set $S\left(v_{4 i}\right)$ contains all $m+2 n$ colors, $i=$ $1,2, \cdots, p$.

The color set $S\left(v_{3 i}\right)$ contains just $m+n+p$ colors from $(i)_{m+2 n}$ to $(m+n+p+i-1)_{m+2 n}$ along cycle $C, i=$ $1,2, \cdots, n$.

The color set $S\left(v_{2 i}\right)$ contains just $m+n+p$ colors which are divided into two parts, the first part is from $(n+i)_{m+2 n}$ to $(n+p+i-1)_{m+2 n}$ along cycle $C$, the second part is from $(m+p+i)_{m+2 n}$ to $(2 m+n+p+i-1)_{m+2 n}$ along cycle $C, i=1,2, \cdots, n$.

Note that $S\left(v_{2 i}\right)$ contains colors from $(n+i)_{m+2 n}$ to $(3 n+$ $p+i-1)_{m+2 n}$ when $m=n, i=1,2, \cdots, n$.

The color set $S\left(v_{1 i}\right)$ contains just $2 n+p$ colors which are divided into three parts, the first part is from $(p+i)_{m+2 n}$ to $(n+p+i-1)_{m+2 n}$ along cycle $C$, the second part is from $(2 n+i)_{m+2 n}$ to $(2 n+p+i-1)_{m+2 n}$ along cycle $C$ and the third part is from $(m+n+p+i)_{m+2 n}$ to $(m+2 n+p+i-$ 1) $m+2 n$ along cycle $C, i=1,2, \cdots, m$.

From the above discussions we can see that:

1. The number of the colors in each color set $C(x)$ is equal to the degree of vertex $x$;

2. The color sets of any two adjacent vertices with the same degree are different.

Thus the resulting coloring is a $(m+2 n)$-AVDPEC of $K_{m, n, n, p}$. The proof is completed.

Theorem 2.2 If $m \geqslant n>p \geqslant 1$, then $\chi_{\mathrm{a}}^{\prime}\left(K_{m, m, n, p}\right)=$ $2 m+n$.

Proof Obviously we have $\chi_{\mathrm{a}}^{\prime}\left(K_{m, m, n, p}\right) \geqslant \Delta\left(K_{m, m, n, p}\right)=$ $2 m+n$. In order to prove $\chi_{\mathrm{a}}^{\prime}\left(K_{m, m, n, p}\right)=2 m+n$, we need only to give the $(2 m+n)$-AVDPEC of $K_{m, m, n, p}$. Suppose the colors that we will use are $1,2, \cdots, 2 m+n$.
We assign color $(i+j-1)_{2 m+n}$ to edge $v_{4 i} v_{3 j}, i=$ $1,2, \cdots, p ; j=1,2, \cdots, n$.

We assign color $(n+i+j-1)_{2 m+n}$ to edge $v_{4 i} v_{2 j}, i=$ $1,2, \cdots, p ; j=1,2, \cdots, m$.

We assign color $(n+m+i+j-1)_{2 m+n}$ to edge $v_{4 i} v_{1 j}$, $i=1,2, \cdots, p ; j=1,2, \cdots, m$.

We assign color $(m+p+i+j-1)_{2 m+n}$ to edge $v_{3 i} v_{2 j}$, $i=1,2, \cdots, n ; j=1,2, \cdots, m$.

We assign color $(p+i+j-1)_{2 m+n}$ to edge $v_{3 i} v_{1 j}, i=$ $1,2, \cdots, n ; j=1,2, \cdots, m$.

We assign color $(m+n+p+i+j-1)_{2 m+n}$ to edge $v_{2 i} v_{1 j}$, $i, j=1,2, \cdots, m$.

We construct a cycle $C$ such that vertex set of $C$ is $2 m+n$ colors $\{1,2, \cdots, 2 m+n\}$ and edge set of $C$ is $\left\{(i)_{2 m+n}(i+\right.$ 1) $2 m+n \mid i=1,2, \cdots, 2 m+n\}$.

The color set $S\left(v_{4 i}\right)$ contains all $2 m+n$ colors, $i=$ $1,2, \cdots, p$.

The color set $S\left(v_{3 i}\right)$ contains just $2 m+p$ colors from $(i)_{2 m+n}$ to $(2 m+p+i-1)_{2 m+n}$ along cycle $C, i=$ $1,2, \cdots, n$.

The color set $S\left(v_{2 i}\right)$ contains just $m+n+p$ colors which are divided into two parts, the first part is from $(n+i)_{2 m+n}$ to $(n+p+i-1)_{2 m+n}$ along cycle $C$, the second part is from $(m+p+i)_{2 m+n}$ to $(2 m+n+p+i-1)_{2 m+n}$ along cycle $C, i=1,2, \cdots, m$.

Note that $S\left(v_{2 i}\right)$ contains colors from $(n+i)_{2 m+n}$ to $(3 n+$ $p+i-1)_{2 m+n}$ when $m=n, i=1,2, \cdots, m$.

The color set $S\left(v_{1 i}\right)$ contains just $m+n+p$ colors which are divided into two parts, the first part is from $(p+i)_{2 m+n}$ to $(n+p+i-1)_{2 m+n}$ along cycle $C$, the second part $(m+$ $n+i)_{2 m+n}$ to $(2 m+n+p+i-1)_{2 m+n}$ along cycle $C$, $i=1,2, \cdots, m$.

From the above discussions we can see that:

1. The number of the colors in each color set $C(x)$ is equal to the degree of vertex $x$;

2. The color sets of any two adjacent vertices with the same degree are different.

Thus the resulting coloring is a $(2 m+n)$-AVDPEC of $K_{m, m, n, p}$. The proof is completed.

Theorem 2.3 If $n>1$, then $\chi_{\mathrm{a}}^{\prime}\left(K_{n, 1,1,1,1}\right)=n+4$.

Proof Obviously we have $\Delta\left(K_{n, 1,1,1,1}\right)=n+3$. By Lemma 1.3, $\chi_{\mathrm{a}}^{\prime}\left(K_{n, 1,1,1,1}\right) \geqslant \Delta\left(K_{n, 1,1,1,1}\right)+1=n+4$. In order to prove $\chi_{\mathrm{a}}\left(K_{n, 1,1,1,1}\right)=n+4$, we need only to give the $(n+4)$ AVDPEC of $K_{n, 1,1,1,1}$. Suppose the colors that we will use are $1,2, \cdots, n+4$.

We assign color $1,2,3$ respectively to edges $v_{51} v_{41}, v_{51} v_{31}$ and $v_{51} v_{21}$.

We assign color $(i+3)_{n+4}$ to edge $v_{51} v_{1 i}(i=1,2, \cdots, n)$. We assign color 3,4 respectively to edges $v_{41} v_{31}$ and $v_{41} v_{21}$.

We assign color $(i+4)_{n+4}$ to edge $v_{41} v_{1 i}, i=1,2, \cdots, n$. We assign color 5 to edge $v_{31} v_{21}$.

We assign color $(i+5)_{n+4}$ to edge $v_{31} v_{1 i}, i=1,2, \cdots, n$. We assign color $(i+6)_{n+4}$ to edge $v_{21} v_{1 i}, i=1,2, \cdots, n$. 
We construct a cycle $C$ such that vertex set of $C$ is $n+4$ colors $\{1,2, \cdots, n+4\}$ and edge set of $C$ is $\left\{(i)_{n+4}(i+\right.$ 1) $\left.{ }_{n+4} \mid i=1,2, \cdots, n+4\right\}$.

The color set $S\left(v_{51}\right)$ contains just $n+3$ colors from $(1)_{n+4}$ to $(n+3)_{n+4}$ along cycle $C$.

The color set $S\left(v_{41}\right)$ contains just $n+3$ colors which are divided into two parts, the first part is $\{1\}$, the second part is from $(3)_{n+4}$ to $(n+4)_{n+4}$ along cycle $C$.

The color set $S\left(v_{31}\right)$ contains just $n+3$ colors which are divided into two parts, the first part is from 2 to 3 along cycle $C$, the second part is from $(5)_{n+4}$ to $(n+5)_{n+4}$ along cycle C.

The color set $S\left(v_{21}\right)$ contains just $n+3$ colors which are divided into two parts, the first part is from 3 to 5 along cycle $C$, the second part is from $(7)_{n+4}$ to $(n+6)_{n+4}$ along cycle C.

The color set $S\left(v_{1 i}\right)$ contains just 4 colors from $(i+3)_{n+4}$ to $(i+6)_{n+4}$ along cycle $C, i=1,2, \cdots, n$.

From the above discussions we can see that:

1. The number of the colors in each color set $C(x)$ is equal to the degree of vertex $x$;

2. The color sets of any two adjacent vertices with the same degree are different.

Thus the resulting coloring is a $(n+4)$-AVDPEC of $K_{n, 1,1,1,1}$. The proof is completed.

Theorem 2.4 If $m>n>1$, then $\chi_{\mathrm{a}}^{\prime}\left(K_{m, n, 1,1,1}\right)=m+$ $n+3$.

Proof Obviously we have $\Delta\left(K_{m, n, 1,1,1}\right)=m+n+2$. By Lemma 1.3, $\chi_{\mathrm{a}}^{\prime}\left(K_{m, n, 1,1,1}\right) \geqslant \Delta\left(K_{m, n, 1,1,1}\right)+1=m+n+$ 3 . In order to prove $\chi_{\mathrm{a}}^{\prime}\left(K_{m, n, 1,1,1}\right)=m+n+3$, we need only to give the $(m+n+3)$-AVDPEC of $K_{m, n, 1,1,1}$. Suppose the colors that we will use are $1,2, \cdots, m+n+3$.

We assign color 1,2 respectively to edges $v_{51} v_{41}, v_{51} v_{31}$. We assign color $(i+2)_{m+n+3}$ to edge $v_{51} v_{2 i}, i=$ $1,2, \cdots, n$.

We assign color $(n+i+2)_{m+n+3}$ to edge $v_{51} v_{1 i}, i=$ $1,2, \cdots, m$.

We assign color 3 to edge $v_{41} v_{31}$.

We assign color $(i+3)_{m+n+3}$ to edge $v_{41} v_{2 i}, i=$ $1,2, \cdots, n$.

We assign color $(n+i+3)_{m+n+3}$ to edge $v_{41} v_{1 i}, i=$ $1,2, \cdots, m$.

We assign color $(i+4)_{m+n+3}$ to edge $v_{31} v_{2 i}, i=$ $1,2, \cdots, n$.

We assign color $(n+i+4)_{m+n+3}$ to edge $v_{31} v_{1 i}, i=$ $1,2, \cdots, m$.

We assign color $(n+i+j+4)_{m+n+3}$ to edge $v_{2 i} v_{1 j}$, $i=1,2, \cdots, n ; j=1,2, \cdots, m$.

We construct a cycle $C$ such that vertex set of $C$ is $m+$ $n+3$ colors $\{1,2, \cdots, m+n+3\}$ and edge set of $C$ is $\left\{(i)_{m+n+3}(i+1)_{m+n+3} \mid i=1,2, \cdots, m+n+3\right\}$.

The color set $S\left(v_{51}\right)$ contains just $m+n+2$ colors from (1) $m+n+3$ to $(m+n+2)_{m+n+3}$ along cycle $C$.

The color set $S\left(v_{41}\right)$ contains just $m+n+2$ colors which are divided into two parts, the first part is $\{1\}$, the second part is from $(3)_{m+n+3}$ to $(m+n+3)_{m+n+3}$ along cycle $C$.
The color set $S\left(v_{31}\right)$ contains just $m+n+2$ colors which are divided into two parts, one part is from 2 to 3 along cycle $C$, other part is from $(5)_{m+n+3}$ to $(m+n+4)_{m+n+3}$ along cycle $C$.

The color set $S\left(v_{2 i}\right)$ contains just $m+3$ colors which are divided into two parts, one part is from $(i+2)_{m+n+3}$ to $(i+$ 4) $m+n+3$ along cycle $C$, other part is from $(n+i+5)_{m+n+3}$ to $(m+n+i+4)_{m+n+3}$ along cycle $C, i=1,2, \cdots, n$.

The color set $S\left(v_{1 i}\right)$ contains just $n+3$ colors from $(n+i+$ $2)_{m+n+3}$ to $(2 n+i+4)_{m+n+3}$ along cycle $C, i=1,2, \cdots, m$.

From the above discussions we can see that:

1. The number of the colors in each color set $C(x)$ is equal to the degree of vertex $x$;

2. The color sets of any two adjacent vertices with the same degree are different.

Thus the resulting coloring is a $(m+n+3)$-AVDPEC of $K_{m, n, 1,1,1}$. The proof is completed.

Theorem 2.5 If $m>n>p>1$, then $\chi_{\mathrm{a}}^{\prime}\left(K_{m, n, p, 1,1}\right)=$ $m+n+p+2$.

Proof Obviously we have $\Delta\left(K_{m, n, p, 1,1}\right)=m+n+p+1$. By Lemma 1.3, $\chi_{\mathrm{a}}^{\prime}\left(K_{m, n, p, 1,1}\right) \geqslant \Delta\left(K_{m, n, p, 1,1}\right)+1=m+$ $n+p+2$. In order to prove $\chi_{\mathrm{a}}^{\prime}\left(K_{m, n, p, 1,1}\right)=m+n+p+2$, we need only to give the $(m+n+p+2)$-AVDPEC of $K_{m, n, p, 1,1}$. Suppose the colors that we will use are $1,2, \cdots, m+n+p+2$. We assign color 1 to edge $v_{51} v_{41}$.

We assign color $(i+1)_{m+n+p+2}$ to edge $v_{51} v_{3 i}, i=$ $1,2, \cdots, p$.

We assign color $(p+i+1)_{m+n+p+2}$ to edge $v_{51} v_{2 i}, i=$ $1,2, \cdots, n$.

We assign color $(p+n+i+1)_{m+n+p+2}$ to edge $v_{51} v_{1 i}$, $i=1,2, \cdots, m$.

We assign color $(i+2)_{m+n+p+2}$ to edge $v_{41} v_{3 i}, i=$ $1,2, \cdots, p$.

We assign color $(p+i+2)_{m+n+p+2}$ to edge $v_{41} v_{2 i}, i=$ $1,2, \cdots, n$.

We assign color $(p+n+i+2)_{m+n+p+2}$ to edge $v_{41} v_{1 i}$, $i=1,2, \cdots, m$.

We assign color $(p+i+j+2)_{m+n+p+2}$ to edge $v_{3 i} v_{2 j}$, $i=1,2, \cdots, p ; j=1,2, \cdots, n$.

We assign color $(p+n+i+j+2)_{m+n+p+2}$ to edge $v_{3 i} v_{1 j}$, $i=1,2, \cdots, p ; j=1,2, \cdots, m$.

We assign color $(2 p+n+i+j+2)_{m+n+p+2}$ to edge $v_{2 i} v_{1 j}$, $i=1,2, \cdots, n ; j=1,2, \cdots, m$.

We construct a cycle $C$ such that vertex set of $C$ is $m+n+$ $p+2$ colors $\{1,2, \cdots, m+n+p+2\}$ and edge set of $C$ is $\left\{(i)_{m+n+p+2}(i+1)_{m+n+p+2} \mid i=1,2, \cdots, m+n+p+2\right\}$.

The color set $S\left(v_{51}\right)$ contains just $m+n+p+1$ colors from $(1)_{m+n+p+2}$ to $(m+n+p+1)_{m+n+p+2}$ along cycle $C$.

The color set $S\left(v_{41}\right)$ contains just $m+n+p+1$ colors which are divided into two parts, the first part is $\{1\}$, the second part is from $(3)_{m+n+p+2}$ to $(m+n+p+2)_{m+n+p+2}$ along cycle C.

The color set $S\left(v_{3 i}\right)$ contains just $m+n+2$ colors which are divided into two parts, the first part is from $(i+1)_{m+n+p+2}$ 
to $(i+2)_{m+n+p+2}$ along cycle $C$, the second part is from $(p+i+3)_{m+n+p+2}$ to $(p+m+n+i+2)_{m+n+p+2}$ along cycle $C, i=1,2, \cdots, p$.

The color set $S\left(v_{2 i}\right)$ contains just $m+p+2$ colors which are divided into two parts, the first part is from $(p+i+1)_{m+n+p+2}$ to $(2 p+i+2)_{m+n+p+2}$ along cycle $C$, the second part is from $(2 p+n+i+3)_{m+n+p+2}$ to $(2 p+m+n+i+2)_{m+n+p+2}$ along cycle $C, i=1,2, \cdots, n$.

The color set $S\left(v_{1 i}\right)$ contains just $n+p+2$ colors from $(p+n+i+1)_{m+n+p+2}$ to $(2 p+2 n+i+2)_{m+n+p+2}$ along cycle $C, i=1,2, \cdots, m$.

From the above discussions we can see that:

1. The number of the colors in each color set $C(x)$ is equal to the degree of vertex $x$;

2. The color sets of any two adjacent vertices with the same degree are different.

Thus the resulting coloring is a $(m+n+p+2)$-AVDPEC of $K_{m, n, p, 1,1}$. The proof is completed.

Theorem 2.6 If $m>n>p>q>1$, then $\chi_{\mathrm{a}}^{\prime}\left(K_{m, n, p, q, 1}\right)$ $=m+n+p+q$.

Proof Obviously we have $\chi_{\mathrm{a}}^{\prime}\left(K_{m, n, p, q, 1}\right) \geqslant \Delta\left(K_{m, n, p, q, 1}\right)$ $=m+n+p+q$. In order to prove $\chi_{\mathrm{a}}^{\prime}\left(K_{m, n, p, q, 1}\right)=$ $m+n+p+q$, we need only to give the $(m+n+p+q)$ AVDPEC of $K_{m, n, p, q, 1}$. Suppose the colors that we will use are $1,2, \cdots, m+n+p+q$.

We assign color $(i)_{m+n+p+q}$ to edge $v_{51} v_{4 i}, \quad i=$ $1,2, \cdots, q$.

We assign color $(q+i)_{m+n+p+q}$ to edge $v_{51} v_{3 i}, i=$ $1,2, \cdots, p$.

We assign color $(q+p+i)_{m+n+p+q}$ to edge $v_{51} v_{2 i}, i=$ $1,2, \cdots, n$.

We assign color $(q+p+n+i)_{m+n+p+q}$ to edge $v_{51} v_{1 i}$, $i=1,2, \cdots, m$.

We assign color $(q+i+j)_{m+n+p+q}$ to edge $v_{4 i} v_{3 j}, i=$ $1,2, \cdots, q ; j=1,2, \cdots, p$.

We assign color $(q+p+i+j)_{m+n+p+q}$ to edge $v_{4 i} v_{2 j}$, $i=1,2, \cdots, q ; j=1,2, \cdots, n$.

We assign color $(q+p+n+i+j)_{m+n+p+q}$ to edge $v_{4 i} v_{1 j}$, $i=1,2, \cdots, q ; j=1,2, \cdots, m$.

We assign color $(2 q+p+i+j)_{m+n+p+q}$ to edge $v_{3 i} v_{2 j}$, $i=1,2, \cdots, p ; j=1,2, \cdots, n$.

We assign color $(2 q+p+n+i+j)_{m+n+p+q}$ to edge $v_{3 i} v_{1 j}$, $i=1,2, \cdots, p ; j=1,2, \cdots, m$.

We assign color $(2 q+2 p+n+i+j)_{m+n+p+q}$ to edge $v_{2 i} v_{1 j}, i=1,2, \cdots, n ; j=1,2, \cdots, m$.

We construct a cycle $C$ such that vertex set of $C$ is $m+$ $n+p+q$ colors $\{1,2, \cdots, m+n+p+q\}$ and edge set of $C$ is $\left\{(i)_{m+n+p+q}(i+1)_{m+n+p+q} \mid i=1,2, \cdots, m+n+p+q\right\}$.

The color set $S\left(v_{51}\right)$ contains just $m+n+p+q$ colors from $(1)_{m+n+p+q}$ to $(m+n+p+q)_{m+n+p+q}$ along cycle $C$.

The color set $S\left(v_{4 i}\right)$ contains just $m+n+p+1$ colors which are divided into two parts, the first part is $\{i\}$, the second part is from $(q+i+1)_{m+n+p+q}$ to $(m+n+p+q+i)_{m+n+p+q}$ along cycle $C, i=1,2, \cdots, q$.
The color set $S\left(v_{3 i}\right)$ contains just $m+n+q+1$ colors which are divided into two parts, the first part is from $(q+i)_{m+n+p+q}$ to $(2 q+i)_{m+n+p+q}$ along cycle $C$, the second part is from $(p+2 q+i+1)_{m+n+p+q}$ to $(p+2 q+m+n+i)_{m+n+p+q}$ along cycle $C, i=1,2, \cdots, p$.

The color set $S\left(v_{2 i}\right)$ contains just $m+p+q+1$ colors which are divided into two parts, the first part is from $(q+$ $p+i)_{m+n+p+q}$ to $(2 p+2 q+i)_{m+n+p+q}$ along cycle $C$, the second part is from $(2 p+2 q+n+i+1)_{m+n+p+q}$ to $(2 p+$ $2 q+m+n+i)_{m+n+p+q}$ along cycle $C, i=1,2, \cdots, n$.

The color set $S\left(v_{1 i}\right)$ contains just $n+p+q+1$ colors from $(q+p+n+i)_{m+n+p+q}$ to $(2 q+2 p+2 n+i)_{m+n+p+q}$ along cycle $C, i=1,2, \cdots, m$.

From the above discussions we can see that:

1. The number of the colors in each color set $C(x)$ is equal to the degree of vertex $x$;

2. The color sets of any two adjacent vertices with the same degree are different.

Thus the resulting coloring is a $(m+n+p+q)$-AVDPEC of $K_{m, n, p, q, 1}$. The proof is completed.

Since $K_{m, n, p, q, r}$ has no two adjacent vertices of maximum degree when $m>n>p>q>r \geqslant 1$, by Lemma 1.4, we may obtain the following Theorem 2.7.

Theorem 2.7 If $m>n>p>q>r \geqslant 1$, then

$$
\chi_{\mathrm{a}}^{\prime}\left(K_{m, n, p, q, r}\right)=m+n+p+q \text {. }
$$

\section{ACKNOWLEDGEMENT}

We would like to thank the referees for their valuable and thoughtful suggestions which greatly improve the present paper. This work was supported by the National Natural Science Foundation of China (Grant Nos. 61163037, 61163054).

\section{REFERENCES}

[1] A. C. Burris and R. H. Schelp, "Vertex-distinguishing proper edgecolorings," J. Graph Theory, vol. 26, no. 2, 1997, pp. 73-82.

[2] O. Favaron, Hao Li and R. H. Schelp, "Strong edge-coloring of graphs," Discrete Mathematics, vol. 159, 1996, pp. 103-109.

[3] C. Bazgan, A. Harkat-Benhamdine, Hao Li and M. Woźniak, "On the vertex-distinguishing proper edge-coloring of graphs," J. Combin Theory(Ser B), vol. 75, 1999, pp. 288-301.

[4] J. Rudašová and R. Soták, "Vertex-distinguishing proper edge colorings of some regular graphs," Discrete Mathematics, vol. 308, 2008, pp. 795802.

[5] P. N. Balister, B. Bollobás and R. H. Schelp, "Vertex-distinguishing coloring of graphs with $\Delta(G)=2$," Discrete Mathematics, vol. 252, no.2, 2002, pp. 17-29.

[6] P. N. Balister, O. M. Riordan and R. H. Schelp," Vertex-distinguishing coloring of graphs," J. Graph Theory, vol. 42, 2003, pp. 95-109.

[7] Zhongfu Zhang, Linzhong Liu and Jianfang Wang, "Adjacent strong edge coloring of graphs," Applied Mathematics Letters, vol. 15, no. 5, 2002, pp. 623-626.

[8] Xinmei Zhao, Xiang'en Chen and Xinsheng Liu, "On the adjacent strong edge coloring of several class of complete 4-partite graphs," Journal of Northwest Normal University(Natural Science), vol. 42, no. 2, 2006, pp. 26-29.

[9] J. A. Bondy and U. S. R. Murty, "Graph Theory with Applications," Macmillan, London and Elsevier, New York, 1976.

[10] Zhongfu Zhang and Jianxun Zhang, "Some sufficient conditions for a graph to be in the first class," Mathematics Journal, vol. 5, no. 2, 1985, pp. $161-165$ 